REVIEW ARTICLE

\title{
Ambulatory Diagnostic-therapeutic Approach for Obstructive Sleep Apnoea Syndrome (OSAS)
}

\author{
J. M. Joshi \\ Department of Respiratory Medicine, T.N. Medical College, BYL Nair Hospital, Mumbai-8 \\ $\ldots \ldots \ldots \ldots \ldots \ldots \ldots \ldots \ldots \ldots \ldots \ldots \ldots \ldots \ldots \ldots \ldots \ldots \ldots \ldots \ldots \ldots \ldots \ldots \ldots \ldots \ldots \ldots \ldots \ldots \ldots \ldots \ldots \ldots \ldots$ \\ Indian J Sleep Med 2008; 3.4, 107-111
}

\section{Introduction}

$S$ leep-disordered breathing (SDB) or sleep apnoea syndromes (SAS) represent a group of conditions that are characterized by an abnormal respiration during sleep. There are three distinct forms of sleep apnea: central, obstructive, and complex (a combination of central and obstructive) In obstructive sleep apnea (OSA) breathing is interrupted by obstruction to airflow despite respiratory effort while in central sleep apnea (CSA) breathing is interrupted by the lack of respiratory effort. In complex sleep apnea (CompSAS), there is a transition from obstructive to central features during the events, often worsened during positive airway pressure treatment. OSA, CompSAS and CSA constitute $84 \% \quad 15 \%$ and $0.4 \%$, of cases respectively ${ }^{(1)}$, making OSA the commonest variety of SAS. Nasal continuous positive airway pressure (CPAP) is the most effective treatment for patients with moderate to severe OSAS. ${ }^{(2,3)}$

The estimated prevalence of obstructive sleep apnea syndrome-OSAS (objective sleeping respiratory disturbance associated with daytime sleepiness) in the United States is $2 \%$ in women and $4 \%$ in men. ${ }^{(4)}$ Epidemiological study from other countries also show that $1-5 \%$ of adult men suffer from OSAS. ${ }^{(5)}$ The prevalence of OSAS in adult Indian population is approximately $3.5 \% .^{(6,7,8)}$ This suggests that in India,

\author{
Address for correspondence \\ Dr. J. M. Joshi \\ Professor and Head \\ Dept of Respiratory Medicine \\ B.Y.L.Nair Ch Hospital, Mumbai 400008 \\ Tel/Fax No: 91-022-23003095 \\ Email: drjoshijm@email.com
}

up to about 34 million people may be suffering from OSAS, which, if diagnosed and treated appropriately, could relieve disabling symptoms related to the disorders. Despite being a common disease, a large number of OSAS cases; an estimated $82 \%$ are not diagnosed. ${ }^{(9)}$

\section{Diagnostic-Therapeutic Algorithm for Obstructive Sleep Apnoea Syndrome}

Full polysomnography (PSG) is currently the "gold standard" for the diagnosis of OSAS and titration of effective continuous positive airway pressure (CPAP). ${ }^{(10,11,12,13,14)}$ PSG provides detailed information on sleep state and respiratory and gas exchange abnormalities, in addition to a range of other variables including body position, heart rate and rhythm, and muscle tone and contraction. ${ }^{(1)}$ Split-night studies are also performed, in which the initial part is devoted to diagnosis but the latter part involves the initiation of CPAP therapy. The following criteria are recommended for the diagnosis of OSAS ${ }^{(11)}$.

A. Excessive daytime sleepiness that is not better explained by other factors

B. Two or more of the following that are not better explained by other factors:

- Choking or gasping during sleep

- Recurrent awakenings from sleep

- Unrefreshing sleep

- Daytime fatigue

- Impaired concentration

C. Overnight monitoring demonstrates five or more obstructed breathing events per hour during sleep. 
These events may include any combination of obstructive apneas/hypopneas or respiratory effortrelated arousals (RERAs)

The patient suspected of OSAS must fulfill criterion A or B, plus criterion C. Further OSAS severity based on the frequency of abnormal respiratory events during sleep is graded as (a) mild : 5-15 events/hour of sleep (b) moderate: 15-30 events/hour of sleep and (c) severe: more than 30 events/hour of sleep.

PSG is time-consuming, requiring 2 nights for at least 6 hours in a sleep laboratory. Limited availability and high cost of PSG is also an important limitation of in-laboratory PSG. In view of the adverse outcomes associated with untreated OSAS, there is an urgent need to evaluate approaches to management that do not unduly rely on sleep laboratory-based PSG studies. ${ }^{(13)}$ Diagnostic-therapeutic approach such as overnight home monitoring using limited PSG (cardio-respiratory variables only) or oximetry in conjunction with ambulatory CPAP titration are alternatives for improved access to diagnostic testing and therapy at least in the initial management of the classic OSAS.

\section{Usual care (PSG obtained before CPAP) or ambulatory management (CPAP without doing PSG).}

A recent study combined portable home monitoring and auto titrating CPAP in the experimental arm, thus obviating the need for a sleep laboratory based PSG. This randomized, controlled, open-label trial $\left.{ }^{(15)}\right]$, found that PSG confers no advantage over the ambulatory approach in terms of diagnosis and CPAP titration in the initial management of OSA. Sixty eight patients with a high pretest probability of moderate to severe OSA by sequential application of the Epworth Sleepiness Scale $(E S S)^{(16)}$, Sleep Apnoea Clinical Score (SACS) ${ }^{(17)}$, and overnight oximetry in the home randomly assigned to usual care (PSG obtained before CPAP) or ambulatory management (CPAP without doing PSG). After 3 months, the PSG and ambulatory groups did not differ in the primary outcome, AHI on CPAP or in the secondary outcomes, ESS score, Sleep Apnea Quality of Life Index (SAQLI), and CPAP. Adherence to CPAP therapy was better in the ambulatory group compared with the PSG group. When resources are scarce, the ambulatory approach provides care for patients most in need of urgent treatment. ${ }^{(15)}$

\section{Ambulatory Testing to Diagnose Obstructive Sleep Apnoea Syndrome Comparable to Polysomnography}

Ambulatory diagnostic algorithms for the OSAS have been previously described. Several portable monitors have been proposed that range in complexity from full PSG to oximetry alone. ${ }^{(18)}$ Monitors that record respiratory variables together with oximetry but without electroencephalography (EEG) and electromyography (EMG) are particularly attractive because the same definition of OSAS as in standard PSG can be employed, and the test can be self-administered by patients in their home, thereby increasing accessibility. ${ }^{(19)}$ Overnight oximetry and portable sleep monitoring at home can identify OSA with a high degree of accuracy. [20,21,22] There is no difference between home- or laboratory based sleep apnea testing on compliance or improvements in sleep apnea-specific quality of life, one of the only verified outcomes of sleep apnea therapy. ${ }^{(19)}$ Exclusion criteria ${ }^{(21)}$ like absence of daytime sleepiness, heart disease, congestive cardiac failure (CCF), cor pulmonale, hypoventilation, stroke, seizures, psychosis and other serious conditions like asthma, hepatic or renal failure should be considered as contraindications to ambulatory sleep apnea testing.

\section{Alternative Methods of Titrating Continuous Positive Airway Pressure}

It has traditionally been recommended that technicians should titrate CPAP pressures overnight in patients with OSA until most of the apnoeas and arousals are abolished, as monitored by PSG. [14] The required pressure is then delivered by a fixed pressure CPAP machine. Overnight PSG based titration is, however, time consuming and labour intensive. Autoadjusting CPAP (auto- CPAP), which adjust pressure according to inspiratory flow limitation; snoring and apnoeas are also used in overnight CPAP titration. ${ }^{(23,24,25)}$ The 95 th centile overnight airway pressure determines the effective level of CPAP required. This pressure may be delivered using a fixed pressure CPAP or patients may use the auto-CPAP machines long term. The auto titration method can be used to initiate CPAP treatment at home or in hospital ${ }^{(26,27,28)}$. Home titration can deliver the same benefits as in-laboratory titration for about two thirds the cost. ${ }^{(29)}$

Another method is to start CPAP by using a formula ${ }^{(30)}$ instead of a formal titration in a sleep 
laboratory. These predict the required CPAP pressure based on neck circumference/ body mass index and oxygen desaturation/ $\mathrm{AHI}^{(31,32,33)}$. West et al ${ }^{(34)}$ randomised prospectively patients diagnosed recently with OSA to one of three different methods of CPAP therapy i.e., (1) long term autotitration, (2) autotitration for 1 week with long term fixed pressure thereafter, and (3) an algorithm method of pressure determination and showed that the method of determining CPAP pressure for treatment of moderate to severe OSA makes no significant difference to clinical outcome measures.

This study showed that although the formula based group $\left(8 \mathrm{cmH}_{2} \mathrm{O}\right)$ was lower than the split-night titration $\left(9.5 \mathrm{cmH}_{2} \mathrm{O}\right)$ pressure, the patients improved equally with respect to sleepiness and adverse effects in both groups. However, the formula is complicated and difficult to use in practice. Another simple method is to put patients on a CPAP pressure based on the body mass index $(\mathrm{BMI})^{(35)}$. The recommended pressures are $8 \mathrm{~cm}$ $\mathrm{H}_{2} \mathrm{O}$ for $\mathrm{BMI}<30 / \mathrm{Kg} \mathrm{m}^{2}, 10 \mathrm{~cm} \mathrm{H}_{2} \mathrm{O}$ between BMI of $30-35 / \mathrm{Kg} \mathrm{m}^{2}$ and $12 \mathrm{~cm} \mathrm{H}_{2} \mathrm{O}$ for $\mathrm{BMI}>35 / \mathrm{Kg} \mathrm{m}^{2}$. Both the algorithm based or arbitrary-pressure pressure approach to determine CPAP pressures for treatment of OSA are simple and equally effective alternatives to the labour intensive overnight PSG based titration or the expensive auto-CPAP titration.

\section{Empiric Auto-adiusting CPAP for Diagnosis And Therapy of OSAS}

Another ambulatory approach is the response to empirical auto-CPAP therapy during 2 weeks in sleepy snorers with clinically suspected OSAS [36]. In this study the CPAP trial predicted the OSAS (AHI 10 events per hour) with positive and negative predictive values of $97 \%$ and $78 \%$, respectively. Furthermore, the trial identified patients using CPAP for more than 4 months who experienced persistent symptomatic improvement with positive and negative predictive values of $92 \%$ and $100 \%$, respectively. Therefore, a CPAP trial may help to diagnose the OSAS, identify patients who benefit from CPAP, and reduce the need for PSG.

\section{Determining Clinical Probability of Obstructive Sleep Apnoea Syndrome}

The ambulatory diagnostic-therapeutic approach requires accurate identification of probable cases of OSAS. Objective sleeping respiratory disturbance associated with daytime sleepiness define the obstructive sleep apnoea syndrome-OSAS. Therefore measures of daytime sleepiness and clinical prediction scores are important to determine probability of OSAS. The Epworth Sleepiness Scale (ESS) [16] is a simple, self-administered questionnaire which is a measure of the probability of falling asleep in a variety of situations i.e. the level of daytime sleepiness. ESS scores correlate significantly with sleep latency measured during the multiple sleep latency test (MSLT) and during overnight PSG. ${ }^{(37)}$ Total ESS scores significantly distinguished normal subjects from patients in various diagnostic groups including obstructive sleep apnea syndrome, narcolepsy and idiopathic hypersomnia. Sleepy snorers are most likely to suffer from OSAS. While the ESS scores of simple snorers do not differ from controls, in patients with OSAS the ESS scores correlate with the respiratory disturbance index and the minimum overnight oxygen saturation. Total ESS scores therefore distinguish patients with primary snoring from those with OSAS. A higher score (up to 24) indicates more sleepiness and correlate with the severity of OSAS. ${ }^{(38)}$ Objective sleepiness can be also be measured by a modification of the Maintenance of Wakefulness test (MWT), a behavioural sleep resistance challenge in which the patient is required to stay awake in a darkened sound protected room. ${ }^{(39)}$ The time taken to fail repeatedly to respond to a visual signal is measured, so a lower score indicates more sleepiness. ${ }^{(40,41)}$

The use of an evidence based clinical prediction tool to accurately assess the pretest probability of OSAS can outperform clinical estimates by sleep specialists ${ }^{(42)}$ In order to help identify the urgency of need for treatment, these clinical features most useful for establishing an accurate estimate of the probability of OSA. The Sleep Apnea Clinical Score SACS ${ }^{(17)}$ is a screening tool based on snoring, witnessed episodes of apnea, neck circumference, and systemic hypertension. A score of 15 or greater gives a likelihood ratio of 4.45 of having moderate to severe OSA. ${ }^{(17)}$ A simple way of using SACS is calculation of adjusted neck circumference i.e. measured neck circumference in $\mathrm{cm}+3 \mathrm{~cm}$ for snoring, $3 \mathrm{~cm}$ for witnessed apnoeas and $4 \mathrm{~cm}$ for systemic hypertension. Adjusted neck circumference of $<43$, between $43-47.9 \mathrm{~cm}$ and $>49 \mathrm{~cm}$ indicate low risk, intermediate risk and high risk for OSAS. ${ }^{(43)}$

\section{Conclusion}

Specialist guidelines favour laboratory-based testing for

Indian Journal of Sleep Medicine (IJSM), Vol. 3, No. 4, 2008 
diagnosis and treatment of sleep apnea; however, this facility is not available to many patients. ${ }^{(43)}$ The high prevalence of OSAS makes it necessary to consider simplified approaches to the diagnosis at least in selected cases $^{(44)}$. Screening for snoring and sleepiness by the ESS can be followed up using a simple risk assessment tool for sleep apnea such as the SACS. Enough evidence now exists that simple ambulatory diagnostic-therapeutic strategies have an equivalent clinical outcome compared with the conventional laboratory-based approach. ${ }^{(43)}$ These simplified strategies should therefore be used where there is inadequate access to PSG. The ambulatory approaches may also be adopted as a first-line strategy for all high-probability OSAS patients. ${ }^{(45,46)}$ Protocols to enhance long term CPAP compliance have been developed and physicians may use experienced CPAP vendors to assist in follow-up to their patients. ${ }^{(43)}$ Patients who have a low sleep apnea probability or who do not respond favorably to CPAP or have difficulties during ambulatory management should be referred to a sleep centre for in-laboratory full PSG. ${ }^{(43,45)}$

\section{References}

1. Morgenthaler TI, Kagramanov V, Hanak V, Decker PA. Complex sleep apnea syndrome: is it a unique clinical syndrome? Sleep. 2006 Sep 1;29(9):1203-9.

2. Sullivan CE, Issa FG, Berthon-Jones M, Eves L. Reversal of obstructive sleep apnoea by continuous positive airway pressure applied through the nares. Lancet $1981 ; 1: 862-5$.

3. Loube DI, Gay PC, Strohl KP, Pack AI, White DP, Collop NA. Indications for positive airway pressure treatment for adult obstructive sleep apnea patients: a consensus statement. Chest 1999;115:863-6.

4. Young T, Palta M, Dempsey J, Skatrud J, Weber S, Badr S. The occurrence of sleep disordered breathing among middle-aged adults. N Engl J Med. 1993; 328:1230-5.

5. Davies RJ, Stradling JR. The epidemiology of sleep apnoea. Thorax 1996; 51 :(Suppl 2): 565-570.

6. Saxena S, Gothi D, Joshi JM. Prevalence of Symptoms and Risk of Sleep Disordered Breathing in Mumbai (India). Indian J Sleep Med 2006; 1.1: 27-31

7. Sharma SK, Kumpawat S, Banga A, Goel A. Prevalence and risk factors of obstructive sleep apnea syndrome in a population of Delhi, India. Chest. 2006;130:149-56.

8. Vijayan VK, Patial K. Prevalence of Obstructive Sleep Apnea Syndrome (OSAS) In Delhi, India. Chest 2006 130: 92S.

9. Young T, Evans L, Finn L, Palta M. Estimation of the clinically diagnosed proportion of sleep apnea syndrome in middleaged men and women. Sleep. 1997;20:705-6.
10. Indications and standards for use of nasal continuous positive airway pressure (CPAP) in sleep apnea syndromes. American Thoracic Society. Official statement adopted March 1944. Am J Respir Crit Care Med. 1994;150:1738-45.

11. Practice Committee of the American Sleep Disorders Association. Practice parameters for the indications for polysomnography and related procedures. Polysomnography Task Force, American Sleep Disorders Association Standards of Practice Committee. Sleep. $1997 ; 20: 406-22$.

12. American Academy of Sleep Medicine Task Force. Sleeprelated breathing disorders in adults: recommendations for syndrome definition and measurement techniques in clinical research. Sleep 1999;22: 667-689.

13. McNicholas WT. Diagnosis of Obstructive Sleep Apnea in Adults. Proc Am Thorac Soc, 2008; Vol 5. pp 154-160.

14. Positive Airway Pressure Titration Task Force of the American Academy of Sleep Medicine. Clinical Guidelines for the Manual Titration of Positive Airway Pressure in Patients with Obstructive Sleep Apnea. Journal of Clinical Sleep Medicine, 2008; 4:157-171.

15. Mulgrew AT, Fox N, Ayas NT, Ryan CF. Diagnosis and initial management of obstructive sleep apnea without polysomnography: a randomized validation study. Ann Intern Med. 2007;146:157-66.

16. Johns MW. A new method for measuring daytime sleepiness: the Epworth sleepiness scale. Sleep 1991;14: 540-5.

17. Flemons WW, Whitelaw WA, Brant R, Remmers JE. Likelihood ratios for a sleep apnea clinical prediction rule. Am J Respir Crit Care Med. 1994;150:1279-85.

18. Ferber $\mathbf{R}$, Millman $\mathbf{R}$, Coppola $M$, et al. ASDA standards of practice: portable recording in the assessment of obstructive sleep apnea. Sleep 1994;17:378-92.

19. Jobin V, Mayer P, Bellemare F. Predictive value of automated oxygen saturation analysis for the diagnosis and treatment of obstructive sleep apnoea in a home-based setting. Thorax $2007 ; 62: 422$.

20. Coppola MP, Lawee M. Management of obstructive sleep apnea syndrome in the home. The role of portable sleep apnea recording. Chest. 1993;104:19-25.

21. Whitelaw WA, Brant R, Flemons WW. Clinical usefulness of home oximetry compared with polysomnography for assessment of sleep apnea. Am J. Respir Crit Care Med. $2000 ; 171: 188-193$.

22. Golpe R, Jime'nez A, Carpizo R. Home sleep studies in the assessment of sleep apnea/hypopnea syndrome. Chest. $2002 ; 122: 1156-61$.

23. Ayas NT, Patel SR, Malhotra A, Schulzer M, Malhotra M Jung $D$, et al. Auto-titrating versus standard continuous positive airway pressure for the treatment of obstructive sleep apnea: results of a meta-analysis. Sleep. 2004; 27 : 249-253.

24. West SD, Jone DR, Stradling JR. Comparison of three ways to determine and deliver pressure during nasal CPAP therapy for obstructive sleep apnea. Thorax. 2006;61:226-231. 
25. Masa JF, Jiménez A, Duran J, Capote F, Monasterio C, Mayos $M$, et al. Alternative methods of titrating continous positive airway pressure. Am J Respir Crit Care Med. 2004;170:1218-1224.

26. Planes C, D'Ortho MP, Foucher A, Berkani M, Leroux $K$, Essalhi $M$, et al. Efficacy and cost of home-initiated Auto- $n$ CPAP versus conventional CPAP. Sleep 2003;26:156-60.

27. Stradling JR, Barbour C, Pitson DJ, Davies RJ. Automatic nasal continuous positive airway pressure titration in the laboratory, patient outcomes. Thorax 1997;52:72-5.

28. Berry RB, Parish JM, Hartse KM. The use of auto-titrating continuous positive airway pressure for treatment of adult obstructive sleep apnea. An American Academy of Sleep Medicine review. Sleep 2002;25:148-73.

29. Stradling JR. Reducing the Cost of Treating Obstructive Sleep Apnea Good News for Patients. Am J Respir Crit Care Med. $2004 ; 170: 1143-4$.

30. Lopez-Campos JL, Garcia Polo C, Leon Jimenez A Gonzalez-Moya E, Arnedillo A, Fernandez Berni JJ. CPAP titration: Different methods for similar clinical results. Eur J Intern Med. 2007;18:230-4.

31. Miljeteig H, Hoffstein V. Determinants of continuous positive airway pressure level for treatment of obstructive sleep apnea. Am Rev Respir Dis. 1993;147:1526-1530.

32. Hoffstein V, Mateika S. Predicting nasal continuous positive airway pressure. Am J Respir Crit Care Med 1994; 150: 486-8.

33. Stradling JR, Hardinge $M$, Smith DM. A novel, simplified approach to starting nasal CPAP therapy in OSA. Respir Med 2004;98:155-8.

34. West SD, Jones DR, Stradling JR. Comparison of three ways to determine and deliver pressure during nasal CPAP therapy for obstructive sleep apnoea. Thorax 2006; $61: 226-231$

35. Hukins CA, Arbitrary-pressure continuous positive airway pressure for obstructive sleep apnea syndrome. Am J Respir Crit Care Med, 2005;171:500-505.

36. Senn O, Brack T, Russi EW, Bloch KE. A continuous positive airway pressure trial as a novel approach to the diagnosis of the obstructive sleep apnea syndrome. Chest. 2006; 129:67-75.

37. Chervin RD, Aldrich MS, Pickett R, Guilleminault C. Comparison of the results of the Epworth Sleepiness Scale and the Multiple Sleep Latency Test. J Psychosom Res. 1997; 42:145-55.

38. Johns MW. Daytime sleepiness, snoring, and obstructive sleep apnea. The Epworth Sleepiness Scale. Chest. $1993 ; 103: 30-6$

39. Bennett LS, Stradling JR, Davies RJO. A behavioural test to assess daytime sleepiness in obstructive sleep apnoea. J Sleep Res 1997;6:142-5.

40. Priest B, Brichard C, Aubert G, Liistro G, Rodenstein DO. Microsleep during a simplified maintenance of wakefulness test. A validation study of the OSLER test. Am J Respir Crit Care Med 2001;163:1619-25.

41. Krieger AC, Ayappa I, Norman RG, Rapoport DM, Walsleben J. Comparison of the maintenance of wakefulness test to a behavioral test-the Oxford sleep resistance (OSLER) test in the evaluation of wakefulness. J Sleep Res $2004 ; 13: 407$

42. Young T, Shahar E, Nieto FJ, Redline S, Newman AB, Gottlieb DJ, et al; Sleep Heart Health Study Research Group. Predictors of sleep-disordered breathing in communitydwelling adults: the Sleep Heart Health Study. Arch Intern Med 2002;162(8):893-900.

43. Skjodt NM. Approach to outpatient management of adult sleep apnea. Can Fam Physician 2008;54:1408-1412

44. ATS/ACCP/AASM Taskforce Steering Committee. Executive summary on the systematic review and practice parameters for portable monitoring in the investigation of suspected sleep apnea in adults. Am J Respir Crit Care Med 2004; 169:1 160-1163.

45. Flemons WW, Whitelaw WA, Brant R, Remmers JE. Likelihood ratios for a sleep apnea clinical prediction rule. Am J Respir Crit Care Med. 1994;150:1279-85.

46. Bloch KE, Senn O, Brack T, Russi EW. Ambulatory Management of Obstructive Sleep Apnea without Polysomnography (Letter). Ann Intern Med. 2007; 147:350. 